\title{
SOME REMARKS ON GROUPS WITH NILPOTENT MINIMAL COVERS
}

\author{
R. A. BRYCE ${ }^{\otimes}$ and L. SERENA \\ (Received 6 December 2006; accepted 4 March 2007) \\ Communicated by E. A. 0'Brien
}

\begin{abstract}
A cover of a group is a finite collection of proper subgroups whose union is the whole group. A cover is minimal if no cover of the group has fewer members. It is conjectured that a group with a minimal cover of nilpotent subgroups is soluble. It is shown that a minimal counterexample to this conjecture is almost simple and that none of a range of almost simple groups are counterexamples to the conjecture.
\end{abstract}

2000 Mathematics subject classification: 20D99.

Keywords and phrases: finite group, cover of a group, almost simple group.

\section{Introduction}

A finite collection of subgroups of a group whose union is the whole group is a cover of the group. The first result on covers seems to have come from Miller [12]. Many different aspects of covers have been studied and applications found in geometry (translation planes) and number theory (for example, Kronecker classes of fields). The reader is referred to the survey article [14] for a discussion of a range of results on covers of groups and some of these applications.

A cover is irredundant if no proper subcollection is also a cover. A minimal cover is irredundant and no collection of subgroups with fewer members is a cover. The earliest results on minimal covers appear in Cohn [7] and Tomkinson [18]. In [4] minimal covers of $\mathrm{GL}_{2}(q)$ and related groups are described. The articles of Maróti [11] and Britnell et al. [3] provide much information about the size of minimal covers of the alternating and symmetric groups, and for a wide class of linear groups. In [9] the sizes of minimal covers for a selection of sporadic simple groups are determined. Every finite group has, of course, an irredundant cover of abelian, even cyclic, subgroups. However the present authors showed in [5] that a group with a minimal cover of abelian subgroups is soluble of very restricted structure. In this note we collect some results

(c) 2009 Australian Mathematical Society 1446-7887/09 \$A2.00+0.00 
on groups that admit a minimal cover with all members nilpotent, a nilpotent minimal cover in short. The nonnilpotent groups in this class can be viewed as generalisations of minimally nonnilpotent groups. We conjecture that groups admitting a nilpotent minimal cover are soluble. As a first step towards a possible proof of this we show, in the next section, that if there is an insoluble group admitting a nilpotent minimal cover there is a finite, almost simple one.

In Section 3 we derive a number of conditions that are necessary in order for a group to admit a nilpotent minimal cover. In Section 4 we limit the range of possible counterexamples to our conjecture by showing that the groups of certain classes of almost simple groups violate these conditions.

For ease of reference we list here two easy lemmas concerning a minimal cover $\mathcal{A}=\left\{A_{1}, \ldots, A_{n}\right\}$ of a group $G$ : proofs are left to the reader.

LEMMA 1.1. If $N \unlhd G$, then either $G=A_{i} N$ for some $i$ or $\left\{A_{i} N / N: 1 \leq i \leq n\right\}$ is a minimal cover for $G / N$.

LeMma 1.2. For $1 \leq i<j \leq n,\left\langle A_{i}, A_{j}\right\rangle=G$.

\section{Minimal counterexample}

Let $G$ be an insoluble group with a nilpotent minimal cover, $\mathcal{A}=\left\{A_{1}, \ldots, A_{n}\right\}$ say. Write $D$ for the intersection of the cover so that $|G: D|$ is finite by a result of Neumann [13]. Since $C:=\operatorname{core}_{G}(D)$ is nilpotent, $G / C$ is insoluble. Moreover, $G / C$ is finite, as it embeds in the symmetric group of degree $|G: D|$ and, by Lemma 1.1, $\left\{A_{i} C / C: 1 \leq i \leq n\right\}$ is a nilpotent minimal cover of $G / C$. We prove more.

PROPOSITION 2.1. If there is an insoluble group admitting a nilpotent minimal cover there is a finite, almost simple one.

We assume that $G$ is a finite, insoluble group of smallest order admitting a nilpotent minimal cover $\left\{A_{1}, A_{2}, \ldots, A_{n}\right\}$. Our proof that $G$ is almost simple begins with two lemmas.

\section{LEMMA 2.2. G is monolithic with nonabelian monolith.}

PROOF. If $W$ is an arbitrary minimal normal subgroup of $G$, then $G / W$ is soluble either because $W A_{i}=G$ for some $i$, and then $G / W \cong A_{i} / A_{i} \cap W$ is nilpotent; or because there is no such $i$ and then $\left\{A_{i} W / W: 1 \leq i \leq n\right\}$ is a nilpotent minimal cover for $G / W$ by Lemma 1.1 so, by the minimality of $G, G / W$ would be soluble. This shows at once that $W$ is not abelian, or $G$ would be soluble. If $X$ is a minimal normal subgroup other than $W$, then $W \cap X=1$ and $G$ embeds in $G / W \times G / X$ which would be soluble, a contradiction. We have therefore proved what was claimed.

Now let $U$ be the monolith of $G$ so that $U=S_{1} \times S_{2} \times \cdots \times S_{m}$ where $S_{1} \cong$ $S_{i}(1 \leq i \leq m)$ and $S_{1}$ is nonabelian and simple. Moreover $\left\{S_{1}, S_{2}, \ldots, S_{m}\right\}$ is a conjugacy class of $G$. If $m=1$, then $G$ is almost simple, so suppose that $m>1$. We denote by $N$ the normaliser in $G$ of $S_{1}$. Note that $U \leq N \neq G$. 
LEMMA 2.3. If $A_{i} \not \leq N$ and $A_{i} \cap S_{1} \neq 1$ then $\left|A_{i}: A_{i} \cap N\right|$ and $\left|A_{i} \cap S_{1}\right|$ are powers of the same prime, $p$ say; and $p^{\prime}$-elements of $A_{i}$ are in $N$.

ProOF. Since $A_{i}$ is nilpotent there is a composition chain from $A_{i} \cap N$ to $A_{i}$ : let $V_{1} / V_{2}$ be one of its factors whose order, a prime, is $p$, say. A $p$-element in $V_{1} \backslash V_{2}$ centralises each $p^{\prime}$-element of $S_{1} \cap A_{i}$ since $A_{i}$ is nilpotent but, on the other hand, conjugates it into an $S_{j}$ whose intersection with $S_{1}$ is trivial. That is $A_{i} \cap S_{1}$ is a p-group. The same argument shows that all factors in the chain above $A_{i} \cap N$ are of this same order $p$. Consequently $\left|A_{i}: A_{i} \cap N\right|$ is a power of $p$. Since $A_{i} \cap N$ is subnormal in $A_{i}$ all $p^{\prime}$-elements of $A_{i}$ are in $N$.

We resume the proof of Proposition 2.1. At most one of the $A_{i}$ is in $N$ by Lemma 1.2; and not all of the $A_{i}$ not in $N$ intersect $S_{1}$ trivially as $S_{1}$ is not contained in an $A_{j}$. We renumber the $A_{i}$, if necessary, so that

$$
A_{i} \not \leq N(1 \leq i \leq n-1) \quad \text { and } \quad A_{i} \cap S_{1} \neq 1(1 \leq i \leq r) \quad \text { where } r \leq n-1 .
$$

Let us assume, for now, that $G / U$ is nilpotent. We write $\mathcal{P}$ for the set of those primes that divide the indices $\left|A_{i}: A_{i} \cap N\right|(1 \leq i \leq r)$; and for $p \in \mathcal{P}$ let $T_{p} / U$ be the Sylow $p$-subgroup of $G / U$. If $p|| A_{i}: A_{i} \cap N \mid$ then $A_{i} \leq T_{p} N$ by Lemma 2.3; note that $T_{p} \unlhd G$ so that $T_{p} N$ is a subgroup. By Lemma 1.2 no two $A_{i}$ are in the same $T_{p} N$ unless $T_{p} N=G$. That is either $|\mathcal{P}|=r$ or $|\mathcal{P}|=1$.

Now

$$
S_{1}=\left(S_{1} \cap A_{1}\right) \cup\left(S_{1} \cap A_{2}\right) \cup \cdots \cup\left(S_{1} \cap A_{r}\right) \cup\left(S_{1} \cap A_{n}\right)
$$

where the first $r$ terms in the union are subgroups of prime-power order either all for the same prime or for $r$ different primes. In the first case choose another prime, $q$ say, dividing $\left|S_{1}\right|$, and then all Sylow $q$-subgroups of $S_{1}$ are in $S_{1} \cap A_{n}$ yielding $S_{1} \leq A_{n}$, a contradiction to the nilpotence of $A_{n}$. In the second case for each $i \in\{1,2, \ldots, r\}$ there is a Sylow subgroup $X_{i}$ of $S_{1}$, not containing $S_{1} \cap A_{i}$, but involving the same prime. Therefore, $X_{i} \leq S_{1} \cap A_{n}$ and, of course, Sylow subgroups of $S_{1}$ for all primes not in $\mathcal{P}$ are all in $S_{1} \cap A_{n}$ so $S_{1} \leq A_{n}$, again a contradiction.

Hence, $G / U$ is not nilpotent and so $U A_{i} \neq G(1 \leq i \leq n)$. The subgroups $U A_{i} / U$ together form a nilpotent minimal cover of $G / U$ so, by the minimality of $G, G / U$ is soluble. The following information about such a group was given in [5, Theorem 11]. Let $Z / U$ be the hypercentre of $G / U$. Then $G / Z$ is monolithic: let $K / Z$ be its monolith, an elementary abelian $t$-group where $t$ is prime. Here $G / K$ is cyclic of order co-prime to $t$. (The group $G / Z$ is Frobenius.) Also $Z=U A_{i} \cap U A_{j}(1 \leq i<j \leq n$ ) and one of the members of this cover is $K / U$; we assume that it is $U A_{n} / U$. The others are, modulo $Z / U$, the complements for $K / U$ in $G / U$. Also note that $U \leq Z \leq U A_{i}$ gives

$$
Z=U\left(Z \cap A_{i}\right) \quad(1 \leq i \leq n) \quad \text { and } \quad Z=(Z \cap N)\left(Z \cap A_{i}\right) \quad 1 \leq i \leq n .
$$

Case 1: $Z \not \leq N$. Then $Z \cap A_{i} \not \leq N(1 \leq i \leq n)$. By Lemma $2.3\left|Z \cap A_{i}: A_{i} \cap Z \cap N\right|$ is a prime power for some prime $p$ and $S_{1} \cap A_{i}$ is a $p$-subgroup whenever $S_{1} \cap A_{i} \neq 1$. 
However (1) shows that

$$
|Z: Z \cap N|=\left|Z \cap A_{i}: A_{i} \cap Z \cap N\right|
$$

so $p$ is the same for all $i$ for which $S_{1} \cap A_{i} \neq 1$, meaning that $S_{1}$ is a union of $p$-subgroups, a contradiction.

Case 2: $Z \leq N$. First we prove a useful lemma.

LEMMA 2.4. Let $H=V L$ be a Frobenius group where the kernel $V$ is elementary abelian and $L$ is a complement for $V$. Assume that $L_{1}, L_{2}$ are proper subgroups of $L$ whose indices in $L$ are co-prime and where $L_{2} \unlhd L$. Let $1 \neq v \in V$. Then $\left\langle L_{1}, L_{2}^{v}\right\rangle=H$.

Proof. Write $T:=\left\langle L_{1}, L_{2}^{v}\right\rangle$. Modulo $V, H=T$ so $T$ acts, by conjugation, irreducibly on $V$. Also $T \cap V \unlhd H$ so, if $T \cap V \neq 1, T=H$, as required. Suppose that $T \cap V=1$. Then $T \cap L \geq L_{1} \neq 1$ so $T=L$ as $H$ is Frobenius and then

$$
\left[L_{2}, v\right] \leq\left\langle L_{2}, L_{2}^{v}\right\rangle \leq L \cap V=1 .
$$

However, $L_{2}$ contains every Sylow subgroup of $L$ for primes dividing $\left|L: L_{1}\right|$ so there is a nontrivial normal subgroup of $L$ with nontrivial centraliser in $V$ contradicting that $L$ acts faithfully and irreducibly on $V$.

Now $N \neq G$ as $m>1$ so no two of $\left\{A_{i} \mid 1 \leq i \leq n-1\right\}$ are in $N$; let us say $A_{i} \not \leq N(1 \leq i \leq n-2)$. We assume that $S_{1} \cap A_{i} \neq 1(1 \leq i \leq s \leq n-2)$; note that $s \geq 2$ as $S_{1}$ is not coverable by three or fewer of the $A_{i} \mathrm{~s}$. By Lemma 2.3 each $\left|A_{i}: A_{i} \cap N\right|(1 \leq i \leq s)$ is a prime power. If two of these indices, say for $i=1,2$, were co-prime then, by Lemma 2.4 and working modulo $Z$,

$$
N \geq\left\langle N \cap A_{1}, N \cap A_{2}\right\rangle=G
$$

which is a contradiction. It follows that

$$
S_{1}=X_{1} \cup X_{2} \cup \cdots \cup X_{s} \cup\left(S_{1} \cap A_{n-1}\right) \cup\left(S_{1} \cap A_{n}\right)
$$

where $X_{j}(1 \leq j \leq s)$ are $p$-groups for the same prime $p$. Note that $p \neq t$. If $U A_{n}=K \not \leq N$, so that $A_{n} \not \leq N$, it follows from Lemma 2.3 that $S_{1} \cap A_{n}$ is a $t$-group (possibly trivial). Now $S_{1}$ is insoluble so, by Burnside's theorem, there is a prime $q$, different from both $p$ and $t$, dividing $\left|S_{1}\right|$. All Sylow $q$-subgroups of $S_{1}$ are therefore in $S_{1} \cap A_{n-1}$ yielding $S_{1} \leq A_{n-1}$ contradicting the nilpotence of $A_{n-1}$. This leaves us to consider $U A_{n}=K \leq N$. However, then $A_{i} \not \leq N(1 \leq i \leq n-1)$ by Lemma 1.2 so, using the argument at the beginning of this paragraph, we find that also $S_{1} \cap A_{n-1}$ is a $p$-group. Consequently all Sylow $q$-subgroups of $S_{1}$ are in $S_{1} \cap A_{n}$ giving $S_{1} \leq A_{n}$, which is another contradiction.

This completes the proof of Proposition 2.1. 


\section{Further reduction}

Next we derive a number of necessary conditions on finite groups admitting a nilpotent minimal cover. These will allow us to qualify further the almost simplicity of a minimum counterexample. Throughout $\mathcal{A}:=\left\{A_{1}, A_{2}, \ldots, A_{n}\right\}$ is a nilpotent minimal cover of a group $G$.

LEMMA 3.1. The intersection of a nilpotent cover for an almost simple group is 1 .

Proof. Let $G$ be almost simple with socle $U$. Now $C_{G}(U)=1$. If $S$ were a Sylow $p$-subgroup of the nontrivial intersection of a nilpotent cover for $G$ it would centralise every $p^{\prime}$-element of $U$ and therefore centralise $U$, which is a contradiction.

The following lemma is well known; it was proved in [12], but we give a proof for the convenience of the reader.

LEMMA 3.2. A finite abelian group with a partition is elementary.

PROOF. Suppose that $G$ is an abelian group with a partition $\left\{B_{i} \mid 1 \leq i \leq n\right\}$. Let $b \in B_{1}$ have prime order $p$. We prove that all elements of $G \backslash B_{1}$ have order $p$. If $a \in G \backslash B_{1}$, say $a \in B_{j}$ where $j>1$, then $a b \notin B_{1} \cup B_{j}$ so $a b \in B_{k}$ where $1 \neq k \neq j$. Now $a^{p}=(a b)^{p} \in B_{j} \cap B_{k}=1$ so all elements outside $B_{1}$ have order $p$ as, similarly, do all elements outside $B_{2}$. Therefore, $G$ has exponent $p$ and is elementary.

PROPOSITION 3.3. Let $G$ be a finite group with trivial centre and a nilpotent minimal cover $\mathcal{A}$. Let $p$ be a prime dividing $|G|$ and suppose that $P \in \operatorname{Syl}_{p}(G)$ is abelian and not normal in $G$. Then:

(a) either $P$ is elementary abelian but not of order $p$; or

(b) $P$ is a Sylow p-subgroup of some $A_{i}$ and

(i) $N_{G}(P)$ is a maximal subgroup of $G$;

(ii) $N_{G}(P)$ is strongly $p$-embedded in $G$ (that is, $\left|N_{G}(P) \cap N_{G}(P)^{g}\right|_{p}=1$ for all $\left.g \in G \backslash N_{G}(P)\right)$;

(iii) and $C_{G}(P)=A_{i}$.

Proof. Suppose that $P$ is in no $A_{i}$; in particular, $P$ is not of order $p$. Using Lemma 1.2

$$
A_{i} \cap A_{j} \cap P \leq Z(G)=1 .
$$

That is, $P$ admits a partition so, by Lemma 3.2, it is elementary.

Next suppose that $P$ is not elementary or is of order $p$. Then $P$ is a Sylow $p$-subgroup of $A_{i}$ for some $i$. If $g \in G$ and $P^{g} \neq P$, then $P^{g}$ is a Sylow $p$-subgroup of $A_{j}$ for some $j \neq i$. Suppose that $N_{G}(P)$ is not a maximal subgroup of $G$ so that $N_{G}(P)<M<G$ for some proper subgroup $M$ of $G$. Choose $g \in M \backslash N_{G}(P)$. Then

$$
M \geq\left\langle N_{G}(P), N_{G}(P)^{g}\right\rangle \geq\left\langle A_{i}, A_{j}\right\rangle=G,
$$


a contradiction so $N_{G}(P)$ is maximal in $G$. Note that $N_{G}(P) \geq A_{i}$. Next note that, for $g \in G \backslash N_{G}(P), N_{G}(P)^{g}=N_{G}\left(P^{g}\right) \geq A_{j}$ for some $j \neq i$. We have

$$
\left|N_{G}(P) \cap N_{G}\left(P^{g}\right)\right|_{p}=1
$$

because, if $1 \neq x \in N_{G}(P) \cap N_{G}(P)^{g}$, with $|\langle x\rangle|=p$, then $C_{G}(x) \geq\left\langle A_{i}, A_{j}\right\rangle=G$, a contradiction to $Z(G)=1$. So $N_{G}(P)$ is strongly $p$-embedded in $G$.

Now we prove that $C_{G}(P)=A_{i}$. Suppose, to obtain a contradiction, that $A_{i}$ is a proper subset of $C_{G}(P)$. There is a $p^{\prime}$-element $x \in C_{G}(P) \backslash A_{i}$. With $1 \neq a \in P$, $a x \notin A_{i}$ so $a x \in A_{j}$ for some $j \neq i$ yielding $a \in A_{j}$ and so $C_{G}(a) \geq\left\langle A_{i}, A_{j}\right\rangle=G$, another contradiction to $Z(G)=1$.

Corollary 3.4. Let $G$ be a finite group with $Z(G)=1, \mathcal{A}$ a nilpotent minimal cover of $G$ and let $P \in \operatorname{Syl}_{p}(G)$ be cyclic, or abelian but not elementary, and not normal in $G$. Then $C_{G}(P)$ is nilpotent and $N_{G}(P)$ is the unique maximal subgroup of $G$ containing $C_{G}(P)$.

Proof. First $P \leq A_{i}$ for some $i$. Let $g \in G \backslash N_{G}(P)$. Then $P^{g} \leq A_{j}$ for some $j \neq i$ and therefore

$$
A_{j}=C_{G}\left(P^{g}\right)=C_{G}(P)^{g}=A_{i}^{g} \text {. }
$$

From this we see that

$$
\left\langle A_{i}, g\right\rangle \geq\left\langle A_{i}, A_{i}^{g}\right\rangle=\left\langle A_{i}, A_{j}\right\rangle=G
$$

Consequently every proper subgroup of $G$ containing $A_{i}$ is contained in $N_{G}(P)$. In other words $N_{G}(P)$ is, as claimed, the unique maximal subgroup of $G$ containing $C_{G}(P)$.

It is this result that allows us to see that various insoluble groups do not admit nilpotent minimal covers. In particular an almost simple group $G$ with an abelian Sylow subgroup $P$ which is cyclic or not elementary does not admit a nilpotent minimal cover if either $C_{G}(P)$ is not nilpotent or if $N_{G}(P)$ is not maximal.

COROLLARY 3.5. With the same hypotheses as in the last corollary each member of $\mathcal{A}$ either contains a Sylow p-subgroup or is a $p^{\prime}$-group; those containing Sylow p-subgroups form a conjugacy class.

Since no group is the union of a conjugacy class of subgroups there are, under these hypotheses, $p^{\prime}$-subgroups in every nilpotent, minimal cover of the group.

\section{Applications}

Here we demonstrate the use of Corollary 3.4 in showing that several classes of potential minimal counterexamples to the solubility of a group with a nilpotent minimal cover are not, in fact, minimally coverable by nilpotent subgroups. 


\subsection{Symmetric groups}

LEMMA 4.1. The alternating groups of degree $n \geq 5$ and the symmetric groups of degree $n \geq 4$ do not have nilpotent minimal covers.

PRoOF. If $P=\langle(123)\rangle \in \mathrm{Syl}_{3}\left(\mathrm{Alt}_{5}\right)$, then $C_{G}(P)=P \leq \mathrm{Sym}_{3} \cap \mathrm{Alt}_{4}$ and consequently there are two maximal subgroups of Alt containing $C_{G}(P)$. This contradicts Corollary 3.4, so Alt 5 does not admit a nilpotent minimal cover. (In any case nilpotent subgroups of Alt5 are abelian so a nilpotent minimal cover would be an abelian minimal cover making Alt 5 soluble by [5].)

Suppose now that $n \geq 6$ and denote $\operatorname{Alt}_{n}$ by $G$. Seeking a contradiction, we suppose that $G$ does have a nilpotent minimal cover. Bertrand's postulate ensures that there is a prime $p$ satisfying $(1 / 2) n<p<n$. Note that $p \geq 5$ and that $2 p>n$ so $p^{2} \nmid|G|$. Let $P=\langle(12 \ldots p)\rangle \in \operatorname{Syl}_{p}\left(\mathrm{Alt}_{n}\right)$. Then, if $H$ is the subgroup of permutations in $\mathrm{Alt}_{n}$ fixing each of $1,2, \ldots, p, C_{G}(P) \leq \operatorname{Alt}_{p} \times H$. Consequently, since

$$
\operatorname{Alt}_{p} \times H<G, \quad \operatorname{Alt}_{p} \leq \operatorname{Alt}_{p} \times H \leq N_{G}(P),
$$

using Corollary 3.4, contradicting the simplicity of Alt $p$.

The proof for the symmetric groups with $n \geq 4$ is similar.

\subsection{Suzuki groups}

Lemma 4.2. None of the Suzuki groups $S z(q)$ has a nilpotent minimal cover.

PROOF. Let $G=S z(q)$ be a finite simple Suzuki group with $q=2^{2 m+1}$ and let $\mathcal{A}=$ $\left\{A_{1}, \ldots, A_{n}\right\}$ be a nilpotent minimal cover of $G$. Let $S \in \operatorname{Syl}_{2}(G)$ and $N=N_{G}(S)$. Then $|N|=q^{2}(q-1)$ and $N$ is a Hall subgroup of $G$ (see [15]). The subgroups of order $q-1$ are cyclic Hall subgroups of $G$. Moreover if $|H|=q-1$, then $C_{G}(y)=H$ for all nonidentities $y$ in $H$. It follows by Proposition 3.3 that $\mathcal{A}$ must contain all subgroups of order $q-1$ among its members. On the other hand, $N$ contains distinct subgroups $H_{1}, H_{2}$ of order $q-1$. If $H_{1} \leq A_{1}, H_{2} \leq A_{2}$, say, then

$$
G=\left\langle A_{1}, A_{2}\right\rangle=\left\langle H_{1}, H_{2}\right\rangle \leq N,
$$

which is a contradiction. Therefore, none of the simple Suzuki groups admits a nilpotent minimal cover.

\subsection{Linear groups}

THEOREM 4.3. Let $\operatorname{PSL}_{n}(q) \leq G \leq \operatorname{PGL}_{n}(q)$ where $n \geq 3$, or $n=2$ and $q \geq 4$. Then $G$ admits no nilpotent minimal cover.

PROOF. We suppose, seeking a contradiction, that some such $G$ does admit a nilpotent minimal cover and produce a contradiction. To this end let $\mathcal{A}=\left\{A_{1}, A_{2}, \ldots, A_{n}\right\}$ be a nilpotent minimal cover of $G$. We write $Z$ for the centre of $\mathrm{GL}_{n}(q)$ and define $\bar{G}$ and $\bar{A}_{i}$, subgroups of $\mathrm{GL}_{n}(q)$, by $\bar{G} / Z=G$ and $\bar{A}_{i} / Z=A_{i}(1 \leq i \leq n)$. Note that 
$\overline{\mathcal{A}}:=\left\{\bar{A}_{i} \mid 1 \leq i \leq n\right\}$ is a nilpotent, irredundant cover of $\bar{G}$. Denote by $V$ the natural vector space on which $\mathrm{GL}_{n}(q)$ acts.

We need in the proof the (seemingly well known) fact that whenever $\operatorname{PSL}_{n}(q)$ is simple, then $G$ satisfying $\operatorname{PSL}_{n}(q) \leq G \leq \operatorname{PGL}_{n}(q)$ is almost simple, so, in particular, its centre is trivial; a proof follows easily from Suzuki [16, Theorem 9.9]. We divide the present proof into cases according to whether $n \geq 4$ or $n<4$.

Case 1: $n \geq 4$. Suppose first of all that $q^{n-1}-1$ has a primitive prime divisor, $p$. Since

$$
q-1=\left(q^{n}-1\right)-q\left(q^{n-1}-1\right)
$$

it follows that $p \nmid q^{n}-1$. Let $V_{1}$ be a subspace of $V$ with dimension one. Write $V=V_{1} \oplus V_{2}$ and let $L$ be the Levi component of this decomposition of $V$ in the stabiliser of the flag $\left(V_{1}, V\right)$ : abstractly $L \cong \mathrm{GF}(q)^{\times} \times \mathrm{GL}\left(V_{2}\right)$.

Now let $\bar{P}$ be the Sylow $p$-subgroup of a Singer cycle of $\operatorname{GL}\left(V_{2}\right)$; on order considerations it is in $\operatorname{SL}\left(V_{2}\right)$. Extend its action to the whole of $V$ via trivial action on $V_{1}$. Order considerations also show that $\bar{P}$ is a Sylow $p$-subgroup of $\operatorname{GL}_{n}(q)$; it is in $\mathrm{SL}_{n}(q)$ and so it is a (cyclic) Sylow subgroup of $\bar{G}$.

If $h \in N_{\bar{G}}(\bar{P})$, then it is easy to see that $V_{1} h, V_{2} h$ both admit the action of $\bar{P}$. However, $V_{1}, V_{2}$ are nonisomorphic as $\bar{P}$-modules so are the unique proper, nontrivial submodules of $\left.V\right|_{\bar{P}}$. Therefore, $V_{1} h=V_{1}$ and $V_{2} h=V_{2}$, so $V_{1}, V_{2}$ are $N_{\bar{G}}(\bar{P})$ submodules of $V$. This shows that $N_{\bar{G}}(\bar{P}) \leq L \cap \bar{G}$.

Now $\bar{G} \not \leq L$ as $\mathrm{SL}_{n}(q) \not L L$. However, Proposition 3.3 requires that $N_{G}(\bar{P} Z / Z)$ be maximal in $G$ and so $N_{\bar{G}}(\bar{P})$ is maximal in $\bar{G}$. Therefore, $N_{\bar{G}}(\bar{P})=L \cap \bar{G}$. However,

$$
\mathrm{SL}\left(V_{2}\right) \leq \mathrm{SL}_{n}(q) \cap L \leq L \cap \bar{G}=N_{\bar{G}}(\bar{P}),
$$

which is a contradiction, since $\bar{P} Z / Z$ is not normal in $G$.

If $q^{n-1}-1$ has no primitive prime divisor then, by Zsygmondy's theorem, $n-1=6$ and $q=2$. That is $G=\mathrm{GL}_{7}(2)$. Here $G$ has a Sylow subgroup $P$ of order 31 whose action splits $V$ as $U \oplus W$ where $\operatorname{dim} U=5$ and on $W$ the action of $P$ is trivial. Therefore, $C_{G}(P)$ contains a copy of $\mathrm{GL}_{2}(2)$ which is not nilpotent, contradicting Corollary 3.4; so Case 1 does not arise.

Case 2: $n \leq 3$. A Singer cycle of $\bar{G}$ is the intersection of $\bar{G}$ with a Singer cycle of $\operatorname{GL}_{n}(q)$. Every Singer cycle of $\bar{G}$ is, of course, in some member of $\overline{\mathcal{A}}$. Denote by $\overline{\mathcal{A}}_{S}$ the subset of $\overline{\mathcal{A}}$ of those members containing a Singer cycle. Also let $\mathcal{T}$ be the set of stabilisers in $\bar{G}$ of one-dimensional subspaces of $V$.

Lemma 4.4. (1) Each $\bar{A} \in \overline{\mathcal{A}}_{S}$ contains exactly one Singer cycle. This Singer cycle has index at most 2 in $\bar{A}$ and if its index is exactly 2 then $n=2$ and $q=2^{\beta}-1$ with $\beta \geq 3$.

(2) We have $\bar{G}=\left(\cup \overline{\mathcal{A}}_{S}\right) \cup(\cup \mathcal{T})$ and no member of $\overline{\mathcal{A}}_{S}$ is omissible from this union. 
Here $\cup \overline{\mathcal{A}}_{S}$ denotes the union of the members of $\overline{\mathcal{A}}_{S}$ and $\cup \mathcal{T}$ the union of the members of $\mathcal{T}$.

ProOf. Let $S$ be a Singer cycle of $\bar{G}$ with $S \leq \bar{A} \in \overline{\mathcal{A}}_{S}$. Suppose firstly that $q^{n}-1$ has a primitive prime divisor, $p$ say. Then, on order considerations, the Sylow $p$-subgroup $\bar{P}$ of $S$ is a Sylow subgroup of $\bar{G}$, even of $\mathrm{SL}_{n}(q)$. Here $\bar{A} \leq C_{\bar{G}}(\bar{P})$ since $\bar{A}$ is nilpotent. As $\bar{P}$ acts irreducibly on $V, S=C_{\bar{G}}(\bar{P})$ which is in $\bar{A}$ so $S=\bar{A}$ confirming (1) in this case.

If, on the other hand, $q^{n}-1$ has no primitive prime divisor then, by Zsygmondy's theorem, $n=2$ and $q=2^{\beta}-1$ for some $\beta \geq 3$. The Sylow 2-subgroups of $\operatorname{SL}_{2}(q)$ and $\mathrm{GL}_{2}(q)$ are generalised quaternion and semidihedral respectively (see Carter and Fong [6, pp. 142-3]) of orders $2^{\beta+1}, 2^{\beta+2}$ so a Sylow 2-subgroup of $\bar{G}$ is one or other of these. $S$ has Sylow 2-subgroup $C$, cyclic of index two in a Sylow subgroup $D$ of $\bar{G}$ and is the unique cyclic subgroup of index 2 in $D$. Here $C$ acts irreducibly on $V$ and so $S \leq C_{\bar{G}}(C) \leq S$, and that is $S=C_{\bar{G}}(C)$. Since $\bar{A}$ is nilpotent $S$ is of index at most 2 in $\bar{A}$. Since a Sylow 2-subgroup of $\bar{G}$ has a unique cyclic subgroup of index 2, $S$ is the only Singer cycle in $\bar{A}$. This completes the proof of (1).

Now the centraliser of an element acting irreducibly on $V$ is a Singer cycle so lies in some $\bar{A} \in \overline{\mathcal{A}}_{S}$. On the other hand, an element whose action on $V$ is reducible is in some member of $\mathcal{T}$. This is obvious when $n=2$ so we may suppose that $g \in \mathrm{GL}_{3}(q)$ acts reducibly on $V$ with $U$ a two-dimensional, irreducible submodule. Write $g=g_{0} g_{1}$ where $g_{0}$ is an $s$-element, supposing $q=s^{\delta}$, and $g_{1}$ an $s^{\prime}$-element. Then $U\left(g_{0}-1\right)$ is a proper $\langle g\rangle$-submodule of $U$ so it is zero; hence, $U$ is irreducible for $\left\langle g_{1}\right\rangle$. Then, by Maschke's theorem, $V=U \oplus W$ where $W$ admits the action of $g_{1}$. However, $W g_{0}$ admits $g_{1}$ so, as the decomposition $\left.V\right|_{\left\langle g_{1}\right\rangle}=U \oplus W$ is unique, $W g_{0}=W$ meaning that $W$ is a one-dimensional subspace of $V$ stabilised by $g$. The nonomissibility of members of $\overline{\mathcal{A}}_{S}$ follows since no Singer cycle stabilises a one-dimensional subspace of $V$. This completes the proof of (2) and with it the proof of Lemma 4.4.

The following corollary comes immediately from Lemma 4.4.

Corollary 4.5. We have $\left|\overline{\mathcal{A}} \backslash \overline{\mathcal{A}}_{S}\right| \leq|\mathcal{T}|$.

We show now that this is false, under our continuing assumption that Theorem 4.3 is not true.

To this end consider all unordered pairs $\sigma=\{U, W\}$ of nonzero, complementary subspaces of $V$. Define an element $a_{\sigma}$ of $\mathrm{SL}_{n}(q)$ as follows (supposing, for convenience, that $\operatorname{dim} U=1$ ): $a_{\sigma}$ is to act completely reducibly on $V$ with $\left.a_{\sigma}\right|_{W}$ being a Singer element of GL(W) and $\left.a_{\sigma}\right|_{U}$ the scalar needed to make det $a_{\sigma}=1$.

Lemma 4.6. If $a_{\sigma}$ is in $\bar{A} \in \overline{\mathcal{A}}$, then $\bar{A} \notin \overline{\mathcal{A}}_{S}$.

Proof. Suppose, in contrast, that $\bar{A} \in \overline{\mathcal{A}}_{S}$. By Lemma 4.4 either $\bar{A}$ is a Singer cycle or contains a unique Singer cycle $S$ with index 2. The first case does not occur as otherwise, by Clifford's theorem, $\left.V\right|_{\left\langle a_{\sigma}\right\rangle}$ would be a direct sum of isomorphic irreducible modules which it is not. In the second case $n=2$ and $a_{\sigma}^{2}$ generates 
the unique subgroup of $S$ of order $(q-1) / 2$. However, this is a subgroup of $Z$, a contradiction since $4 \nmid q-1$.

LEMMA 4.7. If $\sigma, \tau$ are distinct pairs of complementary subspaces of $V$, then there is no member of $\overline{\mathcal{A}}$ containing both $a_{\sigma}$ and $a_{\tau}$.

Proof. Suppose, in contrast, that $a_{\sigma}, a_{\tau} \in \bar{A}$, a member of $\overline{\mathcal{A}}$, and write $H:=$ $\left\langle a_{\sigma}, a_{\tau}\right\rangle$. First suppose that $\left.V\right|_{H}$ is irreducible. Since $\left.V\right|_{\left\langle a_{\sigma}\right\rangle}$ is reducible, and since $H$ is nilpotent, there is a composition factor $K / L$ of $H$ for which $a_{\sigma} \in L,\left.V\right|_{L}$ is reducible whilst $\left.V\right|_{K}$ is not. By Clifford's theorem $\left.V\right|_{L}$ is completely reducible and, since $\left.V\right|_{\left\langle a_{\sigma}\right\rangle}$ has unique decomposition $U \oplus W$, this is also a decomposition of $\left.V\right|_{L}$. Moreover, $U, W$ are conjugate submodules for $L$, a contradiction if $n=3$.

So suppose that $n=2$. Let $|K: L|=r$, a prime. The elements of $\operatorname{ker}_{L}(U)$ and $\operatorname{ker}_{L}(W)$ all have determinant one so they are the identity. Consequently $L$ is cyclic, indeed $L=\left\langle a_{\sigma}\right\rangle$ since its order is $q-1$, the largest possible. Here $W=U \lambda$ for some $r$-element $\lambda \in K \backslash L$. Since $a_{\sigma}$ acts as different scalars $\ell^{-1}, \ell$ on $U, W$, respectively, $\lambda, a_{\sigma}$ do not commute. Writing $U=\mathrm{GF}(q) u$ :

$$
\left(u a_{\sigma}^{-1}\right) \lambda=(\ell u) \lambda=(u \lambda) a_{\sigma}=u\left(\lambda a_{\sigma} \lambda^{-1}\right) \lambda
$$

whence $u=u\left(\lambda a_{\sigma} \lambda^{-1} a_{\sigma}\right)$. That is $\lambda a_{\sigma} \lambda^{-1} a_{\sigma}=1$ so $\lambda a_{\sigma} \lambda^{-1}=a_{\sigma}^{-1}$. However, then $\lambda^{2}$ and $a_{\sigma}$ commute entailing $r=2$ or else $\lambda$ and $a_{\sigma}$ commute. Also the nilpotence of $K$ demands $q-1=2^{\gamma}$ for some $\gamma \geq 2$. The order of $\operatorname{SL}_{2}(q)$ is therefore $2^{\gamma+1}\left(2^{\gamma-1}+1\right)\left(2^{\gamma}+1\right)$ so $K$ is a Sylow 2-subgroup of $\operatorname{SL}_{2}(q)$ and therefore of $H$ also. Here $K$ is a generalised quaternion when $\gamma>2$. In this case $\left\langle a_{\sigma}\right\rangle$ is the unique cyclic subgroup of index 2 in the unique Sylow 2-subgroup of $H$ and, similarly, so is $\left\langle a_{\tau}\right\rangle$. Therefore, $\left\langle a_{\sigma}\right\rangle=\left\langle a_{\tau}\right\rangle$ whence $\sigma=\tau$. When $\gamma=2, G$ is either $\mathrm{PSL}_{2}(5) \cong \mathrm{Alt}_{5}$ or $\mathrm{PGL}_{2}(5) \cong \mathrm{Sym}_{5}$ but in neither case does $G$ have a nilpotent minimal cover, by Lemma 4.1.

It remains to treat the case when $\left.V\right|_{H}$ is reducible. We show first that it is completely reducible. Let us write $q=s^{\delta}$ where $s$ is a prime. It will be enough to show that $s \nmid|H|$, that is, that $O_{s}(H)=1$ since $\bar{A}$ is nilpotent. Suppose that $X$ is a proper, nonzero submodule of $\left.V\right|_{H}$. Then $V / X$ is irreducible for $H$ since it is irreducible for $\left\langle a_{\sigma}\right\rangle$. Therefore, $O_{s}(H)$ is in the kernel of both $X, V / X$ but not in the kernel of $V$. If $1 \neq h \in O_{s}(H), v X \mapsto v(h-1)$ is a well-defined $\left\langle a_{\sigma}\right\rangle$-isomorphism $V / X \rightarrow X$, which is a contradiction. Hence, $O_{s}(H)=1$ so, by Maschke's theorem, $\left.V\right|_{H}=X \oplus Y$ for some $H$-submodule $Y$. However, $X$ and $Y$ admit both $a_{\sigma}, a_{\tau}$ each of which gives a unique splitting of $V$. Therefore, $\sigma=\tau$. The proof of Lemma 4.7 is complete.

There are more unordered pairs of complementary subspaces of $V$ than there are subspaces of dimension one. Hence, $\left|\overline{\mathcal{A}} \backslash \bar{A}_{S}\right|>|\mathcal{T}|$, which provides a contradiction to Corollary 4.5. Thus, our assumption that Theorem 4.3 was false is incorrect and Theorem 4.3 is established. 
4.4. Sporadic simple groups In this section we give references from the literature to show that the sporadic simple groups do not have a nilpotent minimal cover. The idea is to indicate in each a Sylow subgroup $P$ of prime order for which either $N_{G}(P)$ is not maximal or $C_{G}(P)$ is not nilpotent so as to invoke Corollary 3.4. In what follows $C_{n}$ indicates a cyclic Sylow subgroup of order $n$ in the group under consideration.

(1) The Mathieu group $G=M_{11}$. Here $|G|=2^{4} \cdot 3^{2} \cdot 5 \cdot 11$ and $N_{G}\left(C_{5}\right)$ is a Frobenius group of order 20 and is not maximal (see [17, p. 211]).

(2) The Mathieu group $G=M_{12}$. Here $|G|=2^{6} \cdot 3^{3} \cdot 5 \cdot 11$ and $N_{G}\left(C_{5}\right)=C_{2} \times F$ where $F$ is a Frobenius group of order 20; it is not maximal (see [17, p. 212]).

(3) The Mathieu group $G=M_{22}$. Here $|G|=2^{7} \cdot 3^{2} \cdot 5 \cdot 7 \cdot 11$ and $N_{G}\left(C_{5}\right)$ is a Frobenius group of order 20 and is not maximal (see [17, p. 212].)

(4) The Mathieu group $G=M_{23}$. Here $|G|=2^{7} \cdot 3^{2} \cdot 5 \cdot 7 \cdot 11 \cdot 23$ and $N_{G}\left(C_{5}\right)$ is a semi-direct product of $C_{15}$ by $C_{4}$ and is not maximal (see [17, p. 213]).

(5) The Mathieu group $G=M_{24}$. Here $|G|=2^{10} \cdot 3^{3} \cdot 5 \cdot 7 \cdot 11 \cdot 23$ and $N_{G}\left(C_{11}\right)$ is a Frobenius group of order 110 and it is not maximal (see [17, p. 213]).

(6) The Janko group $G=J_{1}$. Here $|G|=2^{3} \cdot 3 \cdot 5 \cdot 7 \cdot 11 \cdot 19$ and $C_{G}\left(C_{5}\right)=C_{5} \times$ $D_{6}$ is the direct product of $C_{5}$ and a dihedral group of order 6 , so it is not nilpotent (see [17, p. 213]).

(7) The Hall-Janko group $G=J_{2}$. Here $|G|=2^{7} \cdot 3^{3} \cdot 5^{2} \cdot 7$ and $N_{G}\left(C_{7}\right)$ is a Frobenius group of order 42 and it is not maximal (see [17, p. 214]).

(8) The Janko group $G=J_{3}$. Here $|G|=2^{7} \cdot 3^{5} \cdot 5 \cdot 7 \cdot 17 \cdot 19$ and $N_{G}\left(C_{17}\right)$ is a Frobenius group of order $17 \cdot 8$ and it is not maximal (see [17, p. 214]).

(9) The Janko group $G=J_{4}$. Here $|G|=2^{21} \cdot 3^{3} \cdot 5 \cdot 7 \cdot 11^{3} \cdot 23 \cdot 29 \cdot 31 \cdot 37 \cdot 43$ and $C_{G}\left(C_{7}\right)=C_{7} \times \operatorname{Sym}_{5}$ is not nilpotent (see [17, p. 215]).

(10) The Conway group $G=C_{3}$. Here $|G|=2^{10} \cdot 3^{7} \cdot 5^{3} \cdot 7 \cdot 11 \cdot 23$ and $C_{G}\left(C_{7}\right) \cong C_{7} \times \mathrm{Sym}_{3}$ is not nilpotent (see [17, p. 216]).

(11) The Conway group $G=C o_{2}$. Here $|G|=2^{18} \cdot 3^{6} \cdot 5^{3} \cdot 7 \cdot 11 \cdot 23$ and $N_{G}\left(C_{11}\right)$ is a Frobenius group of order 110 (see [17, p. 216]); it is not maximal (see [8, p. 154]).

(12) The Conway group $G=C o_{1}$. Here $|G|=2^{21} \cdot 3^{9} \cdot 5^{4} \cdot 7^{2} \cdot 11 \cdot 13 \cdot 23$ and $C_{G}\left(C_{11}\right)=C_{11} \times \operatorname{Sym}_{3}$ is not nilpotent (see [1, p. 302]).

(13) The Fischer group $G=F_{22}$. Here $|G|=2^{17} \cdot 3^{9} \cdot 5^{2} \cdot 7 \cdot 11 \cdot 13$ and $C_{G}\left(C_{7}\right) \cong$ $C_{7} \times \mathrm{Sym}_{3}$ is not nilpotent (see [2, p. 251]).

(14) The Fischer group $G=F_{23}$. Here $|G|=2^{18} \cdot 3^{13} \cdot 5^{2} \cdot 7 \cdot 11 \cdot 13 \cdot 17 \cdot 23$ and $C_{G}\left(C_{13}\right) \cong C_{13} \times \operatorname{Sym}_{3}$ is not nilpotent (see [2, p. 252]).

(15) The Fischer group $G=F_{24}^{\prime}$. Here $|G|=2^{21} \cdot 3^{16} \cdot 5^{2} \cdot 7^{3} \cdot 11 \cdot 13 \cdot 17 \cdot 23 \cdot 29$ and $C_{G}\left(C_{13}\right) \geq C_{13} \times \mathrm{Sym}_{3}$ so is not nilpotent (see [2, p. 252]).

(16) The Baby Monster $G=F_{2}$. Here

$$
|G|=2^{41} \cdot 3^{13} \cdot 5^{6} \cdot 7^{2} \cdot 11 \cdot 13 \cdot 17 \cdot 19 \cdot 23 \cdot 31 \cdot 47
$$

and $C_{G}\left(C_{11}\right) \cong C_{11} \times \mathrm{Sym}_{5}$ is not nilpotent (see [8, p. 217]). 
(17) The Fischer group (Monster) $G=F_{1}$. Here

$$
|G|=2^{46} \cdot 3^{20} \cdot 5^{9} \cdot 7^{6} \cdot 11^{2} \cdot 13^{3} \cdot 17 \cdot 19 \cdot 23 \cdot 29 \cdot 31 \cdot 41 \cdot 47 \cdot 59 \cdot 71
$$

and $C_{G}\left(C_{23}\right) \cong C_{23} \times \operatorname{Sym}_{4}$ is not nilpotent (see [8, p. 234]).

(18) The Higman-Sims group $G=H S$. Here $|G|=2^{9} \cdot 3^{2} \cdot 5^{3} \cdot 7 \cdot 11$ and $N_{G}\left(C_{7}\right)$ is a Frobenius group of order 42 and it is not maximal (see [17, p. 220]).

(19) The Held group $G=H e$. Here $|G|=2^{10} \cdot 3^{3} \cdot 5^{2} \cdot 7^{3} \cdot 17$ and $N_{G}\left(C_{17}\right)$ is a Frobenius group of order $17 \cdot 8$ and it is not maximal (see [17, p. 221]).

(20) The Suzuki group $G=\operatorname{Suz}$. Here $|G|=2^{13} \cdot 3^{7} \cdot 5^{2} \cdot 7 \cdot 11 \cdot 13$ and $C_{G}\left(C_{7}\right) \geq$ $C_{7} \times \mathrm{Alt}_{4}$ is not nilpotent (see [1, p. 303]).

(21) The McLaughlin group $G=M c$. Here $|G|=2^{7} \cdot 3^{6} \cdot 5^{3} \cdot 7 \cdot 11$ and $N_{G}\left(C_{11}\right)$ is not maximal (see [8, p. 100]).

(22) The Lyons group $G=L y$. Here $|G|=2^{8} \cdot 3^{7} \cdot 5^{6} \cdot 7 \cdot 11 \cdot 31 \cdot 37 \cdot 67$ and $C_{G}\left(C_{7}\right) \simeq C_{7} \times \mathrm{SL}_{2}(3)$ is not nilpotent (see [17, p. 223]).

(23) The Rudvalis group $G=R u$. Here $|G|=2^{14} \cdot 3^{3} \cdot 5^{3} \cdot 7 \cdot 13 \cdot 29$ and $N_{G}\left(C_{29}\right)$ is a Frobenius group of order $29 \cdot 14$ (see [17, p. 224]) and it is not maximal (see [8, p. 126]).

(24) The O'Nan-Sims group $G=O^{\prime} N$. Here $|G|=2^{9} \cdot 3^{4} \cdot 5 \cdot 7^{3} \cdot 11 \cdot 19 \cdot 31$ and $N_{G}\left(C_{11}\right)$ has order 110 (see [17, p. 225]) and it is not maximal (see [8, p. 132]).

(25) The Thompson group $G=T h$. Here $|G|=2^{15} \cdot 3^{10} \cdot 5^{3} \cdot 7^{2} \cdot 13 \cdot 19 \cdot 31$ and $N_{G}\left(C_{19}\right)$ is a Frobenius group of order $19 \cdot 18$ (see [17, p. 225]) and it is not maximal (see [10, p. 79]).

(26) The Harada group $G$. Here $|G|=2^{14} \cdot 3^{6} \cdot 5^{6} \cdot 7 \cdot 11 \cdot 19$ and $C_{G}\left(C_{7}\right)=C_{7}$ $\times \mathrm{Alt}_{5}$ is not nilpotent (see $[17$, p. 226]).

\section{References}

[1] M. Aschbacher, Sporadic Groups, Cambridge Tracts in Mathematics, 104 (Cambridge University Press, Cambridge, 1994).

[2] - 3-Transposition Groups, Cambridge Tracts in Mathematics, 124 (Cambridge University Press, Cambridge, 1997).

[3] J. Britnell, A. Evssen, R. M. Guralnick, P. E. Holmes and A. Maróti, 'Sets of elements that pairwise generate a linear group', J. Combin. Theory Ser. A 115 (2008), 442-465.

[4] R. A. Bryce, V. Fedri and L. Serena, 'Subgroup coverings of some linear groups', Bull. Aust. Math. Soc. 60 (1999), 227-238.

[5] R. A. Bryce and L. Serena, 'A note on minimal coverings of groups by subgroups', J. Aust. Math. Soc. 71 (2001), 159-168.

[6] R. Carter and P. Fong, 'The Sylow 2-subgroups of the finite classical groups', J. Algebra 1 (1964), 139-151.

[7] J. E. Cohn, 'On n-sum groups', Math. Scand. 75 (1994), 45-58.

[8] J. H. Conway, R. T. Curtis, S. D. Norton, R. A. Parker and R. A. Wilson, An ATLAS of Finite Groups (Oxford University Press, Oxford, 1985).

[9] P. E. Holmes, 'Subgroup coverings of some sporadic simple groups', J. Combin. Theory Ser. A 113 (2006), 1204-1213.

[10] S. A. Linton, 'The maximal subgroups of the Thompson group', J. London Math. Soc. (2) 39 (1989), 79-88. 
[11] A. Maróti, 'Covering symmetric groups with proper subgroups', J. Combin. Theory Ser. A $\mathbf{1 1 0}$ (2005), 97-111.

[12] G. A. Miller, 'Groups in which all operations are contained in a series of subgroups such that any two have only the identity in common', Bull. Amer. Math. Soc. 12 (1906), 445-449.

[13] B. H. Neumann, 'Groups covered by finitely many cosets', Publ. Math. Debrecen 3 (1954), 227-242.

[14] L. Serena, 'On finite covers of groups by subgroups', in: Advances in Group Theory 2002, Proceedings of the Intensive Bimester Dedicated to the Memory of Reinhold Baer (eds. F. de Giovani and M. L. Newell) (Aracne, Roma, 2003).

[15] M. Suzuki, 'On a class of doubly transitive groups', Ann. of Math. 75 (1962), 105-145.

[16] Group Theory I (Springer, Berlin, 1982).

[17] S. A. Syskin, 'Abstract properties of the simple sporadic groups', Russian Math. Surveys 35 (1980), 209-246.

[18] M. J. Tomkinson, 'Groups as the union of proper subgroups', Math. Scand. 81 (1994), 191-198.

R. A. BRYCE, Department of Mathematics, The Australian National University, Canberra, ACT 0200, Australia

e-mail: bryce@maths.anu.edu.au

L. SERENA, Dipartimento di Matematica e Applicazioni per l'Architettura, Universitá degli Studi di Firenze, piazza Ghiberti 27, 50122 Firenze, Italy e-mail: luigi.serena@unifi.it 